\title{
Feature Extraction and Classification of Heart Sounds Signals Based on Time- Dependent Entropy and Spectral Entropy Estimation
}

\author{
Rosario Ríos-Prado ${ }^{1}$, Álvaro Anzueto-Ríos ${ }^{1}$, Blanca Tovar-Corona ${ }^{1}$ \\ ${ }^{1}$ Instituto Politécnico Nacional (UPIITA-IPN), Mexico City, Mexico
}

\begin{abstract}
In this paper, two entropy methods based on Shannon Entropy are exploited, the Time-Dependent Entropy and the Spectral Entropy, calculated in a time domain and frequency domain, respectively. The two calculated entropies together with the Probability Distribution were obtained from a database that contains simultaneous recordings from the four main auscultation areas. These areas are used to test if the probability of detecting the abnormality increases in any of the heart valves and to compare the results in each area respect to signals randomly selected from the database. The parameters obtained from 20 randomly selected signals of the data were used as input features for the K-Nearest Neighbour classifier, obtaining accuracies of $90 \%$ and $80 \%$ for pathologic and normal sounds classification, respectively.

Finally, the features calculated from all the databases were separated and presented in each auscultation area in a $3 D$-graph where a visible separability is shown. Results suggest that some noise associated with valve dysfunction is reflected in the entropy values. Besides, results show that information in each area is different and the analysis of the four areas might improve the classification when there is a pathology.
\end{abstract}

\section{Introduction}

According to the World Health Organization (WHO), cardiovascular diseases are the leading cause of death in the world. Nowadays, there are sophisticated techniques that help physicians to diagnose the functioning of the heart. However, these might not be available to the whole population in public services or patients must wait long periods of time to have their tests $[1,2,3,4]$. For this reason, it is necessary to provide auxiliary signal processing techniques for the detection of abnormalities in the cardiac valves as it has been addressed in other works [2,3]. phonocardiography (PCG) and electrocardiography (ECG) are low cost and non-invasive techniques that can help to monitor the mechanical activity and electrical activity of the heart, respectively [3,4]. As shown in Figure 1, PCG is a technique used to record the mechanical activity of the heart where the main sounds, $\mathrm{S} 1$ and $\mathrm{S} 2$, and murmurs can be detected $[4,5,6]$.

In this work, a simultaneous recording and analysis of four main auscultation areas (aortic, pulmonary, tricuspid, and mitral) are proposed, where two goals are pursued: 1) to test if the use of four channels increases the probability of detecting the abnormality in any of the heart valves, and, 2) to test if there is any difference of the extracted parameters on each auscultation areas.

\section{Normal heart sound and window segmentation}

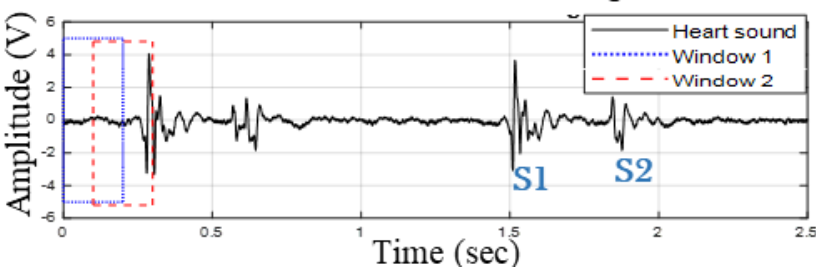

Figure 1. A. PCG signal with window segmentation. B. ECG signal simultaneously recorded with PCG.

As it has been proposed in previous works $[3,4,7]$, the variations that exist between the normal and abnormal heart sounds allow to calculate features that can be distinguished and used in automatic classification. However, classification techniques must be accompanied by signal processing techniques to clean the raw signals, making possible the segmentation of heart sounds into cycles and then identifying $\mathrm{S} 1$ and $\mathrm{S} 2$ to find abnormalities.

In Section 2, the procedure to extract features in the PCG recordings and classification of sounds into normal and pathologic is presented. It is also presented the PCG database description. Section 3 shows the results of the methodology and, Section 4 and 5 exposes a discussion and conclusion, respectively.

\section{Methodology}

In this section, the feature extraction of three parameters and classification of normal and pathologic heart sounds from the PCG database are described.

\subsection{Database}


The PCG database belongs to a set of signals that include normal and abnormal sounds which were acquired at $2000 \mathrm{~Hz}$, using the electronic system described in [2]. The sound signals have been cut in segments of 10 seconds, making a total of 480 segments, separated in sets of five signals, containing the four auscultation areas sounds and the ECG signal. The characteristics of this database are described in Table 1, where the kind of pathologies is shown.

The database was used raw, with no digital filtering and the parameters were extracted for each of the auscultation areas. Besides, 20 signals were selected randomly from the whole set of the four auscultation areas, 10 normal and 10 pathologic, to be classified and the results are compared to the classification carried out per auscultation area.

Table 1. Description of segments in each class.

\begin{tabular}{lcc}
\hline Description of the database. & & \\
\hline Subject & Segments & Age (years) \\
\hline Normal & 240 & $22-31(24.15 \pm 2.13)$ \\
\hline Aortic regurgitation & 98 & \\
Aortic stenosis & 52 & $16-77(59.6 \pm 25.15)$ \\
Mitral regurgitation & 48 & \\
Tricuspid regurgitation & 32 & \\
\hline
\end{tabular}

\subsection{Time-Dependent Entropy (TDE)}

The TDE described in $[8,9,10]$ has been used in the characterization of different biological signals, such as EEG and ECG. The analysis of the TDE proposed in [8], carries out an estimation of the uncertainty between the discrete values of the signal with the calculus of the Shannon Entropy. The estimation of the signal $x(t)$ is calculated using shifting time windows, defined in Equation (1), with size $w$ and shifting $d$, where $x_{i}$ is the discrete values of the signal with probabilities $p_{i}, m$ indicates the index of each window, $p_{i}^{m}$ is the Probability of the Mass Function (PMF) per window and $L$ is the number of windows. The result of the associated entropy to each probability is defined in Equation (2).

$$
\begin{gathered}
W(m ; w, d)=\{x(k), k=1+m d, \ldots, w+m d\} \\
H(m)=-\sum_{i=1}^{L} p_{i}^{m} * \log \left(p_{i}^{m}\right)
\end{gathered}
$$

Where it must be fulfilled $w \leq \frac{K(K-w)}{d} \in N$ y $M=$ $\frac{K-w}{d}$. From $m=0$ up to the last window $m=\frac{K-w}{d}$.

\subsection{Spectral Entropy (SE)}

The SE technique, introduced in [11], is an estimation that uses Power Spectral Density (PSD) as a function of Probability Density and, alike the TDE, it is carried out through shifting windows as defined in Equation (1). The $\mathrm{SE}$ has been used to describe different time series as proposed in $[11,12]$, used to describe sleep stages or epileptic seizure in ECG signals, providing satisfactory results because of the bandwidth of such events is well defined and relatively narrow. However, in PCG signals, the frequencies cover a wider range and the normal events such as S1 and S2 are overlapped in frequency with the abnormal, such as murmurs. To calculate the SE, the Discrete Fourier Transform (DFT) is used. Then, the PSD is calculated with the Equation (3) and the $P_{k}^{m}$ with Equation (4). Finally, the SE is calculated with Equation (5) for each window $w_{k}$, where $P_{k}^{m}$ represents the PMF, $m$ the window index and $p_{k}$ its probability.

$$
\begin{gathered}
S^{m}\left(w_{k}\right)=\left|X^{m}\left(w_{k}\right)\right|^{2} \\
P_{k}^{m}=\frac{S^{m}\left(w_{k}\right)}{\sum_{j=1}^{w} S^{m}\left(w_{j}\right)} \\
S E(m)=-\sum_{k=1}^{L} P_{k}^{m} \log \left(P_{k}^{m}\right)
\end{gathered}
$$

\subsection{Feature extraction}

The procedure of feature extraction is presented in Figure 2. Three parameters are extracted from the raw PCG signals, one in the time domain and two more in the frequency domain and they are used for the classification. The preprocessing step consists of the window segmentation of the signals, then these segments follow two different paths: 1) time-domain analysis with TDE and 2) frequency domain analysis with SE and PD. In the second step, the entropies were calculated for each window of a signal and for both estimations the window duration was defined as 0.2 seconds with an overlap of 0.1 seconds between them, as shown in Figure 1 with a solid line and dotted line tagged as Window 1 and 2, respectively. The mean of the TDE for all windows is taken as one of the input parameters for the classification. Whereas in the frequency domain two parameters are extracted: the mean of the SE for all windows and the mean of dominant values of each window of the PD taken from SE analysis as is shown in Figure 2.

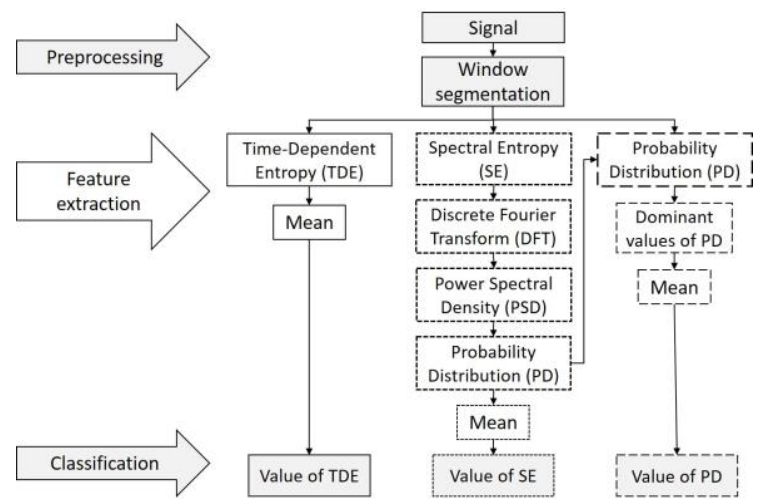

Figure 2. Diagram of PD, TDE and SE extraction from heart sound. 


\section{Results}

The result of the entropy calculus obtained from the shifting windows is depicted in Figure 3 with red lines, in left and right columns for normal and pathologic, respectively.

According to the TDE technique, the largest values are obtained when the differences in consecutive amplitudes increases, that means, when the data changes abruptly. In this way, the S1 and S2 events are enhanced in the major changes of amplitude as is observed in Figure 3A. Unlike the normal sound, the pathologic has some noise associated with valve dysfunction and this is reflected in the entropy values. From Figure 3B, it is possible to deduce that the differences between the amplitudes are more uniform than normal sound, it means that the entropy in the pathologic signal presents constant changes but they are not sudden. Therefore, it is not possible to identify if the peaks correspond to $\mathrm{S} 1$ or $\mathrm{S} 2$, contrary to the situation in the normal signal.

Opposite of TDE, SE analyses the time series as a statistic distribution that, at the same time gives, as a result, a signal whose amplitude is non-dependent on the input signal amplitude, as observed in Figures 3C and 3D.

A. Normal heart sound and TDE B. Abnormal heart sound and TDE
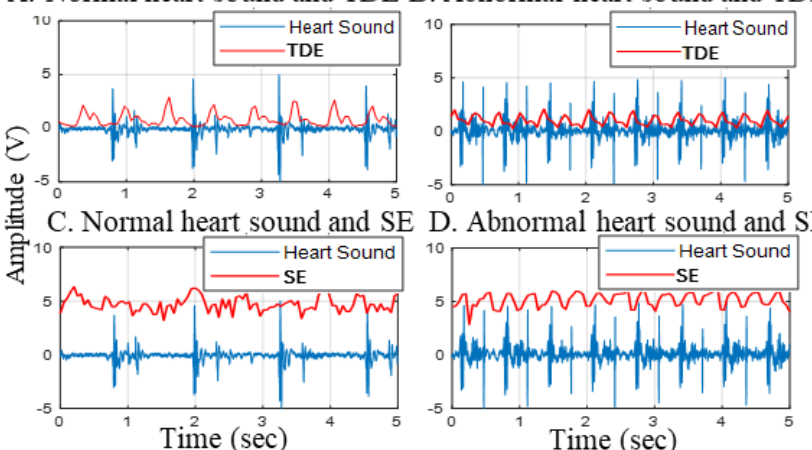

Figure 3. A. Normal sound and Dependent-Time Entropy, B. Abnormal sound and Dependent-Time Entropy, C. Normal sound and Spectral Entropy, D. Abnormal sound and Spectral Entropy.

As it can be seen, TDE has related straight away with the time series shape, but the SE depends on the distribution of the PD, which can be interpreted as normalized Shannon Entropy value, where the amplitude value in the time series does not affect the final result of SE. From Figure 3, it is observed a slight difference between one feature and the other, however, when quantitative analysis is carried out, the results of TDE, SE and PD are significant, so the values for the normal and pathologic signals can be used as classification parameters.

Figure 4 shows a 3D graph from the three parameters extracted, where each axis corresponds to one parameter. Each point represents one of the 20 segments randomly selected. These results show that the variations in the signals and the changes in their entropies, provide ranges that allow the separation of the two classes. A classification has been done using the Nearest Neighbours algorithm $(\mathrm{KNN})$ with $\mathrm{k}=4$ nearest neighbours and the number of classes is two. The input parameters and classification results are shown in Figure 4 where the elements inside the circles represent the classified data. The accuracy percentage obtained in the classification for the 20 randomly selected segments of signals is $90 \%$ for the pathologic and $80 \%$ for the normal.

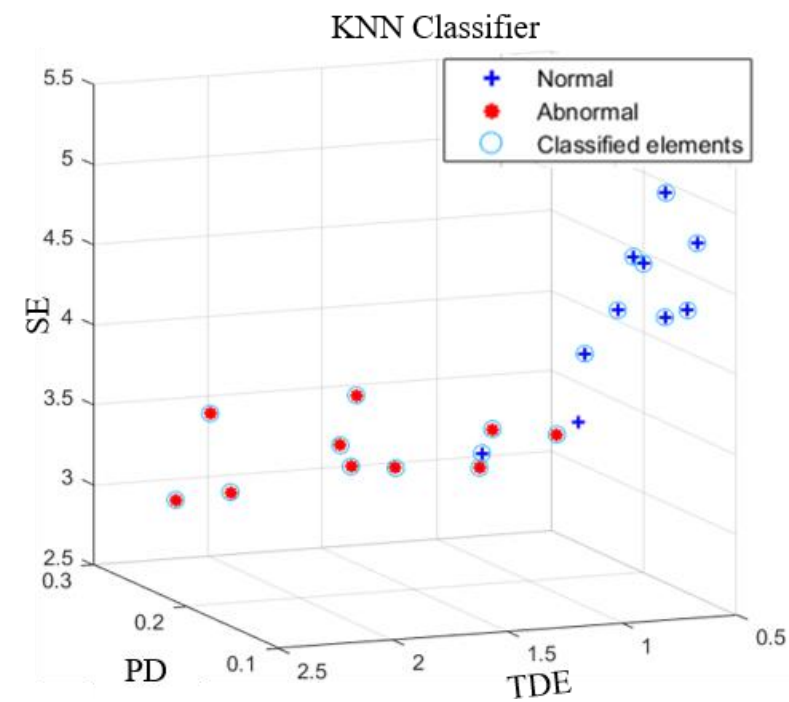

Figure 4. K-NN Classifier for random signal selection.

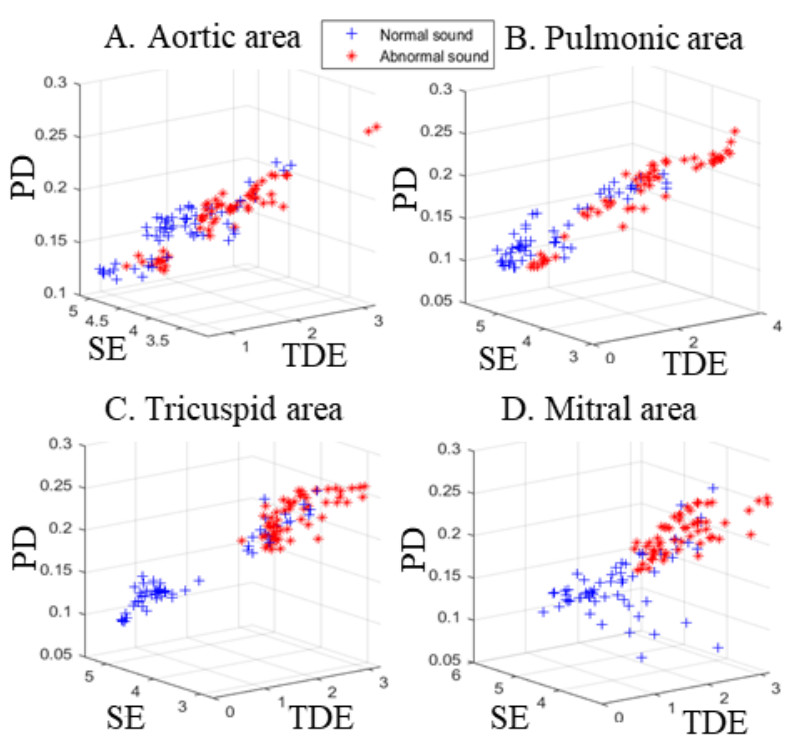

Figure 5. 3D graphs for each auscultation area with the extracted parameters: PD, TDE and SE.

According to the graphs in Figure 5, the separability exists for the calculated parameters, however, some data cannot be classified correctly since their nearest neighbours belong to the other class. This phenomenon might be happening for two reasons: 1) In presence of 
pathologies that affect, for instance, the mitral valve the recording on the aortic area is the less useful to describe the murmur since it is the furthest position from the mitral valve, which can be verified in Figure 5. That is, not all the auscultation areas provide the same information and a pathologic sound can be classified as normal if the wrong position is analysed; 2) the entropy values are calculated on the raw signal, without previous digital pre-processing to attenuates noise. This cleaning process was not carried out since the bandwidth of the main sounds is partially overlapped with that of the murmurs.

\section{Discussion}

The results shown in Figure 5, give the premise that the multidimensional analysis can still be explored with different signal processing and classification techniques.

Comparing to previous works, the processing techniques describe here do not require pre-processing, however, if applied, they might improve the separability results.

Work has to be done considering multidimensional techniques and to compare the results of each auscultation area depending on the pathology. But the results shown here give an approximation for the detection of auxiliary automatic detection of valve abnormalities with a low-cost system to be applied in first-level hospitals were cardiologist is not available.

The methodology for automatic classification of sounds into normal and pathologic was described and results are presented. The TDE, SE and PD are proposed as input parameters for the classification technique KNN and the use of SE had not been reported before in the analysis of heart sound signals.

\section{Conclusion}

Results show that the data can be separated using this methodology and comparing results when analysing the 4 areas recorded simultaneously promising results to show that they provide more information and might improve the correct classification since not all areas provide the same information for all the pathologies. Therefore, the methodology can be used in education to assess auscultation abilities by using it as auxiliary support.

The classification presents $90 \%$ of efficiency for the 20 segments randomly selected. However, it is necessary to test it in a larger database and to analyse results on each auscultation area for different pathologies. Therefore, the preliminary results demonstrate that recording the four auscultation areas simultaneously provide more information than only one area showing that the information extracted is different on each auscultation area, which indicates that the pursued objectives are correct and further exploration will improve the classification efficiency.

\section{Acknowledgements}

To CONACYT Mexico for the scholarship granted for Master Studies to Rosario Rios Prado.

To the Biomechanics Laboratory and Instrumentation and Signal Processing Laboratory, both at UPIITA, IPN.

\section{References}

[1] S. Babaei, and A. Geranmayeh, "Heart Sound Reproduction Based on Neural Network Classification of Cardiac Valve Disorders Using Wavelet Transforms of PCG Signals," Computers in biology and medicine, vol. 39 no. 1, pp. 8-15, 2009.

[2] R. R. Prado, A. A. Ríos, and B. T. Corona, "Methodology to Discern Between Non-Pathological Sound from Regurgitation and Aortic Stenosis, Using DTW," Revista de la Facultad de Ciencias (UNAL), vol. 8, no. 1, p. 138-155, Jan. 2019.

[3] R. R. Prado, A. A. Ríos, and B. T. Corona, "Análisis de Señales de Sonido Cardiaco Empleando la Técnica DTW para Discernir entre Señales No Patológicas y Regurgitación Aórtica," Memorias del Congreso Nacional de Ingeniería Biomédica, vol. 5, no. 1, p. 202205, Nov. 2018

[4] I. Maglogiannis et al., "Support Vectors Machine-based Identification of Heart Valve Diseases Using Heart Sounds," Computers methods and programs in biomedicine, vol. 95, no.1, pp. 47-61, Jul. 2009.

[5] A. G. Tilkian, and M. Boudreau, "Areas of Auscultation, Understanding Heart Sounds and Murmurs," W. B. Saunders Company: pp. 28-151. 1993.

[6] A. Sbrollini et al., "Second Heart Sound Onset to Identify T-Wave Offset," Computing in Cardiology, vol. 44, pp. 1-4, Sep. 2017.

[7] H. Moghaddasi, F. Almasganj, and A. Zoroufian, "Imaging of Heart Acoustic Based on the Sub-space Methods Using a Microphone Array," Computer Methods and Programs in Biomedicine, vol. 146, pp. 1-22, Jul. 2017.

[8] A. Bezerianos, S. Tong, and N. Thakor, "Time-Dependent Entropy Estimation of EEG Rhythm Changes Following Brain Ischemia," Annals of Biomedical Engineering, vol. 31, no. 2, pp. 221-232, Feb. 2003.

[9] M.E. Torres, M.M. Añino, G. Schlotthauer, "Automatic Detection of Slight Parameter Changes Associated to Complex Biomedical Signals Using Multiresolution Q-entropy," Medical Engineering \& Physics, vol. 25, no. 10, pp. 859-867, Dec. 2003.

[10] S. Farashi, "A Multiresolution Time-Dependent Entropy Method for QRS Complex Detection," Biomedical Signal Processing and Control, vol. 24, pp. 63-71, Feb. 2016.

[11] T. Inouye, et al., "Quantification of EEG Irregularity by Use of the Entropy of the Power Spectrum," Electroencephalography and Clinical Neurophysiology, vol. 79, no. 3, pp. 204-210, Sep. 1991.

[12] A. N. Bermúdez, E. M. Spinelli, and C. M Muravchik, "Detección de Eventos en Señales de EEG Mediante Entropía Espectral,” XVIII Congreso Argentino de Bioingeniería, Sep. 2011.

Address for correspondence:

Rosario Ríos Prado.

Laboratorio de Instrumentación y Procesamiento de Señales. Unidad Profesional Interdisciplinaria en Ingeniería y Tecnologías Avanzadas (UPIITA-IPN),

Av. Instituto Politécnico Nacional No. 2580, Col Barrio la Laguna Ticomán, Ciudad de México, C.P. 07340.

E-mail address: r.riosp27@gmail.com 